\title{
The CORALIE survey for southern extra-solar planets
}

\section{The return of the giant planet orbiting HD 192263^}

\author{
N. C. Santos ${ }^{1,2}$, S. Udry ${ }^{1}$, M. Mayor ${ }^{1}$, D. Naef ${ }^{1}$, F. Pepe ${ }^{1}$, D. Queloz ${ }^{1}$, G. Burki ${ }^{1}$, N. Cramer ${ }^{1}$, and B. Nicolet ${ }^{1}$ \\ 1 Observatoire de Genève, 51 ch. des Maillettes, 1290 Sauverny, Switzerland \\ 2 Centro de Astronomia e Astrofísica da Universidade de Lisboa, Observatório Astronómico de Lisboa, Tapada da Ajuda, \\ 1349-018 Lisboa, Portugal
}

Received 16 April 2003 / Accepted 15 May 2003

\begin{abstract}
The presence of a planet around the K dwarf HD 192263 was recently called into question by the detection of a periodic photometric signal with the same period as the one observed in radial velocity. In this paper, we investigate this possibility, using a combination of radial-velocity, photometry, and bisector measurements obtained simultaneously. The results show that while the observed radial-velocity variation is always very stable in phase, period, and amplitude, the photometric signal changes with time. The combined information strongly suggests that the observed radial-velocity variation is being produced by the presence of a planet, as firstly proposed. The photometric variations are either not connected to the planetary companion, or can eventually be induced by the interaction between the planet and the star. Finally, the radial-velocity data further show the presence of a long term trend, whose origin, still not clear, might be related to the presence of another companion to the system.
\end{abstract}

Key words. planetary systems - stars: individual: HD 192263

\section{Introduction}

Radial-velocity techniques have brought to light more than 100 planetary candidates around solar-type stars ${ }^{1}$. The odd properties of many of the exoplanet candidates raised some scepticism from the community. The first exoplanet discovered, orbiting the solar-type star 51 Peg (Mayor \& Queloz 1995), is itself a good example. Its particularly short-period orbit ( 4.23 days) led some astronomers to cast doubts about its existence: e.g. Gray (1997) suggested that the radial-velocity variations were due to non-radial pulsations rather than to the presence of a planetary mass companion. Later on, this result was withdrawn (Gray 1998) and the presence of the planet around 51 Peg confirmed.

Other similar examples exist in the literature. Exploring the fact that the radial-velocity technique only gives us the minimum mass for the companion, Han et al. (2001) suggested that the planetary candidates were in fact low mass stars on orbits seen edge-on. This result was easily refuted by statistical

Send offprint requests to: N. C. Santos,

e-mail: nuno.santos@oal.ul.pt

* Based on observations collected at the La Silla Observatory, ESO (Chile), with the CORALIE spectrograph at the 1.2-m Euler Swiss telescope and at the La Palma Observatory, Spain, with the P7 photometer at the 1.2-m MERCATOR Belgian telescope.

${ }^{1}$ See e.g. http://obswww . unige.ch/Exoplanets arguments in the case of random orbital-plane inclinations (Halbwachs et al. 2000; Jorissen et al. 2001; Pourbaix 2001; Pourbaix \& Arenou 2001). Again, the "planetary origin" of the radial-velocity variations was then considered to be the best one.

It is known that the radial-velocity technique is not sensitive only to the motion of a star around the center of mass of a star/planet system. Intrinsic variations, such as non-radial pulsation (Brown et al. 1998), inhomogeneous convection or spots, are expected to induce radial-velocity variations (e.g. Saar \& Donahue 1997; Saar et al. 1998; Santos et al. 2000b; Paulson et al. 2002; Tinney et al. 2002). These situations can prevent us from finding planets (if the perturbation is larger than the orbital radial-velocity variation) or give us false candidates (if they produce a periodic signal over a few rotational periods). A good example of this effect is given by the periodic radial-velocity variation observed for the dwarf HD 166435 , that was shown to be due to a spot rather than to the presence of a planetary companion (Queloz et al. 2001).

The presence of unknown stellar blends can also induce spurious radial-velocity signals, which can "simulate" the presence of a planetary companion in the case of triple systems. An example is given by HD 41004 in which the moving spectrum of a faint spectroscopic binary companion induces a planetarytype signature on the primary star (Santos et al. 2002; Zucker et al. 2003). 
Table 1. Stellar parameters for HD 192263.

\begin{tabular}{lcc}
\hline \hline Parameter & Value & Reference \\
\hline Spectral type & K2V & colour-index; $M_{v}$ \\
Parallax [mas] & $50.27 \pm 1.13$ & Hipparcos \\
Distance [pc] & 19.9 & Hipparcos \\
$m_{v}$ & 7.79 & Hipparcos \\
$B-V$ & 0.938 & Hipparcos \\
$M_{v}$ & 6.30 & - \\
Luminosity $\left[L_{\odot}\right]$ & 0.34 & Flower (1996) \\
Mass $\left[M_{\odot}\right]$ & 0.75 & Santos et al. (2003) \\
$\log R_{\mathrm{HK}}^{\prime}$ & -4.56 & Henry et al. (2002) \\
$v \sin i\left[\mathrm{~km} \mathrm{~s}{ }^{-1}\right]$ & 1.99 & CORALIE \\
& & Santos et al. (2002) \\
$T_{\text {eff }}[\mathrm{K}]$ & $4995 \pm 50$ & Santos et al. (2003) \\
{$[\mathrm{Fe} / \mathrm{H}]$} & $0.04 \pm 0.05$ & Santos et al. (2003) \\
$\log g$ & $4.76 \pm 0.15$ & Santos et al. (2003) \\
\hline
\end{tabular}

In this context, another planetary companion that was recently called back into question is the case of HD 192263. The star was announced to harbor a Jupiter-mass planetary companion on a 24-day period orbit (Santos et al. 2000a; Vogt et al. 2000). Recently however, Henry et al. (2002) have detected a photometric variation with a period compatible with the period observed in the radial-velocity data. The authors have then concluded that the planet around this star was no longer needed to explain the radial-velocity signal, and that the case of HD 192263 was similar to the one observed in HD 166435.

In this study, we analyze in more details the situation concerning HD 192263. Long term and simultaneous radialvelocity, bisector, and photometric measurements are presented $^{2}$. The results show that the presence of a planet is still needed to explain the observed radial-velocity signal. The sporadic observed photometric variations can eventually be explained as the result of interactions between the planet and the host star. In Sect. 2 we review the stellar parameters of HD 192263, and in Sect. 3 we present the available radial-velocity data. In Sect. 4 we analyze the combined radialvelocities, bisector, and photometric data, exploring the different possibilities to explain the observations. We conclude in Sect. 5 .

\section{Stellar parameters}

The basic stellar parameters of HD 192263 (HIP99711, BD -01 3925, ADS 13547 A) have been discussed in detail in Santos et al. (2000a). These are recalled and updated in Table 1, where we have included new spectroscopic determinations of the atmospheric parameters (Santos et al. 2003) and of $v \sin i$ (Santos et al. 2002). A new $\log R_{\mathrm{HK}}^{\prime}$ value is also quoted from Henry et al. (2002).

\footnotetext{
2 The radial-velocity and photometry measurements are available in electronic form at the CDS via anonymous ftp to cdsarc.u-strasbg.fr $(130.79 .128 .5)$ or via http://cdsweb.u-strasbg.fr/cgi-bin/qcat?J/A+A/406/373
}
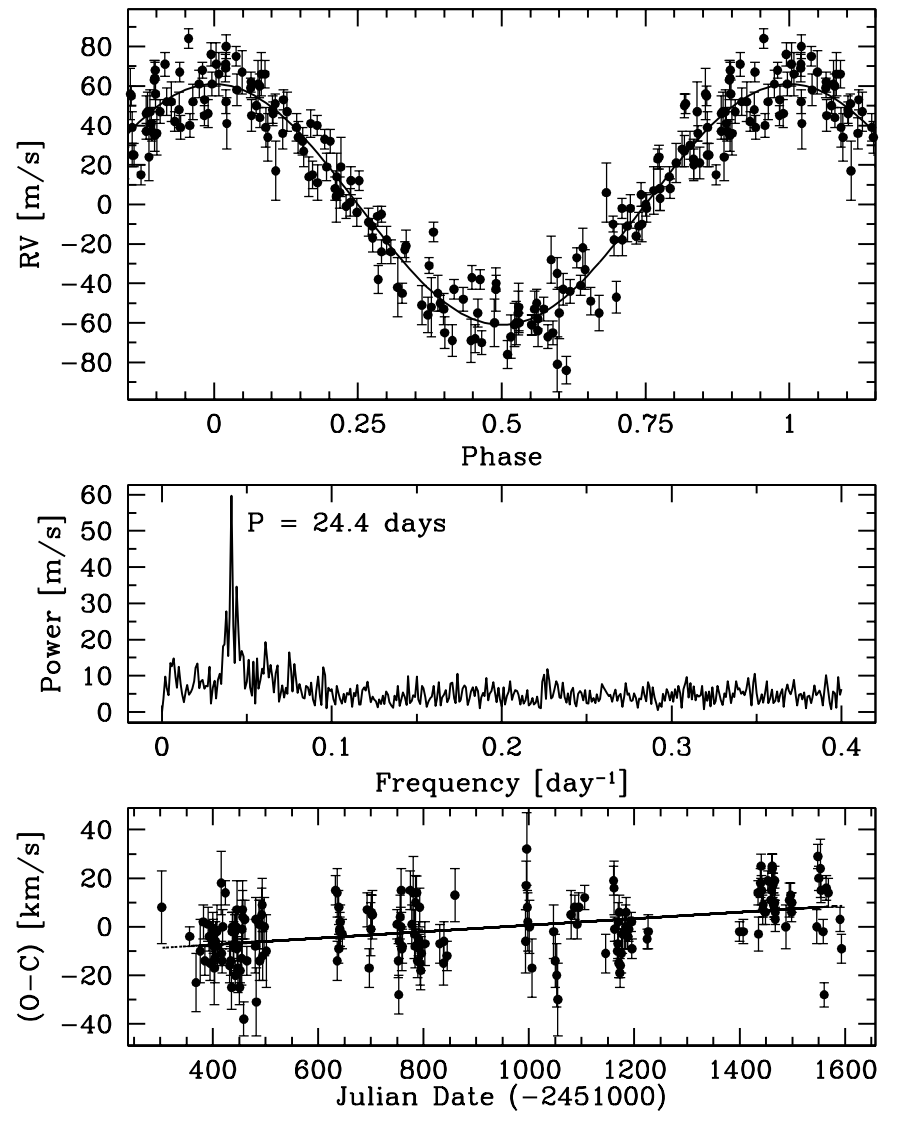

Fig. 1. Upper panel: phase-folded diagram of the radial velocities of HD 192263. The solid curve represents the best Keplerian fit. Middle panel: periodogram of the radial velocities, showing a very well defined peak at the observed 24 .4-day period. Lower panel: residuals of the 24.4-day orbital solution, showing the presence of a long term trend in the data. The line represents a linear fit, and has a slope of $4.8 \pm 0.8 \mathrm{~m} \mathrm{~s}^{-1} \mathrm{yr}^{-1}$.

\section{Radial velocities}

HD 192263 is part of the Geneva extra-solar planet search programme with the CORALIE spectrograph (on the 1.2-Swiss telescope, La Silla, ESO, Chile; Udry et al. 2000). In this context, it was found to present a periodic radial-velocity signal, interpreted as an indication of the presence of a planetary mass companion orbiting this $\mathrm{K}$ dwarf star (Santos et al. 2000a). Before discussing in the next sections the origin of the observed radial-velocity variations, let us first simply consider the planetary explanation.

Since the planet discovery paper, we have been continuously adding radial velocities of this star, using the CORALIE spectrograph, gathering so a total of 182 observations. The velocities were computed using a weighted cross-correlation mask (Pepe et al. 2002b), which permitted to effectively reduce the rms of our measurements.

As before, an analysis of the radial velocities shows the presence of a signal with a period of about 24 days and an amplitude of $61 \mathrm{~m} \mathrm{~s}^{-1}$. In Fig. 1 we plot a phase-folded diagram as well as a Fourier transform (FT) of the radial velocities. In Fig. 2 we show a time series of the observed radial velocities for the whole period of our measurements. The two plots clearly 

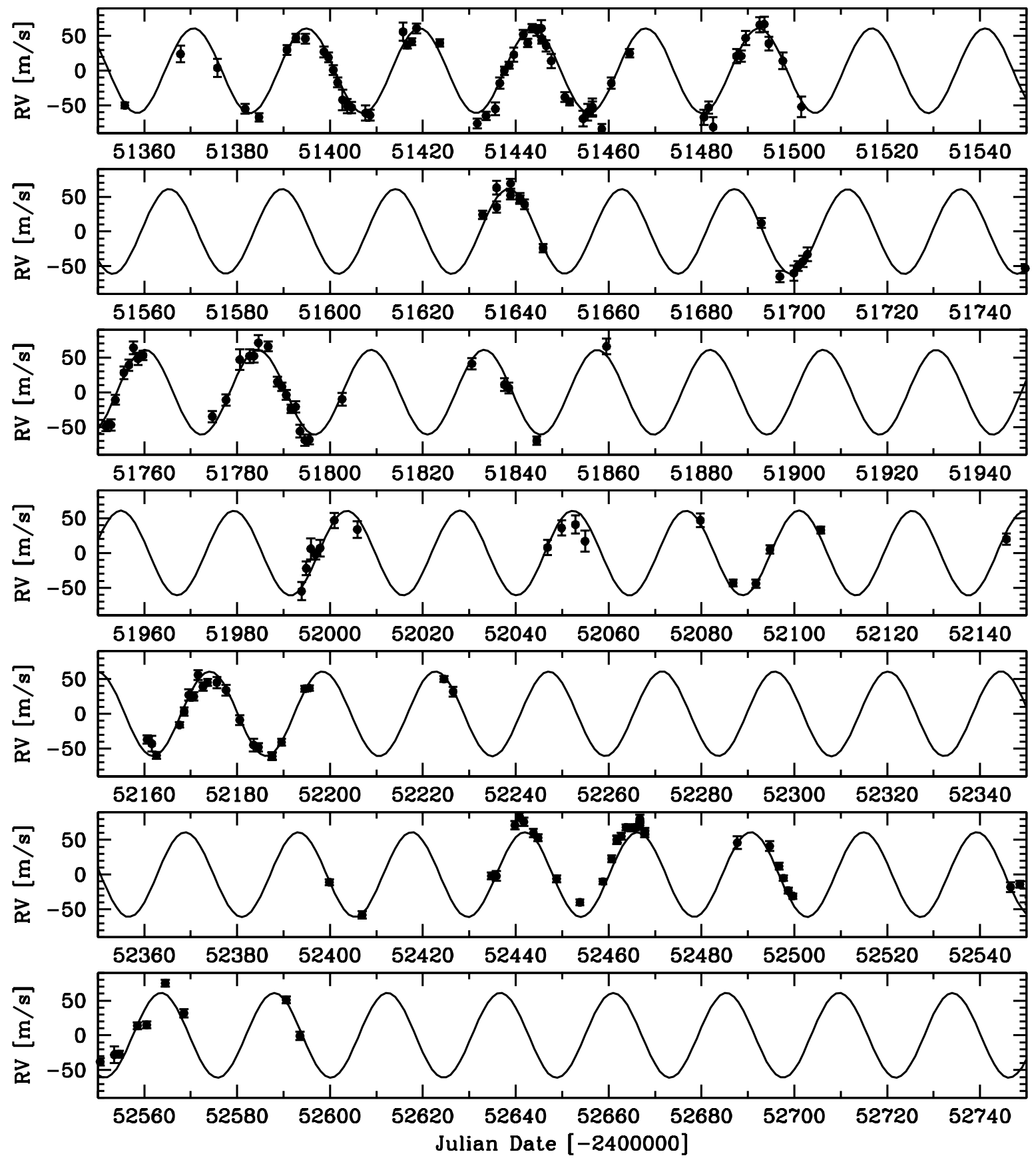

Fig. 2. Radial-velocity time series of HD 192263 for the complete span of our measurements. The curve represents the fitted orbital solution. It is interesting to see the long-term phase stability of the radial-velocity signal.

show the presence of a very stable periodic radial-velocity signal that can be interpreted as the signature of a $0.72 M_{\text {Jup }}$ planetary companion orbiting HD 192263 every 24.348 days, on a (quasi-)circular orbit. The separation is about $0.15 \mathrm{AU}$. The inferred planetary orbital parameters and minimum mass are listed in Table 2.

Considering the stellar rotational period of 24.5 days found by Henry et al. $(2002)^{3}$, and knowing that the $v \sin i$ of

${ }^{3}$ A value compatible with the measured activity level of the star, that implyes a $P_{\text {rot }} \sim 21$ days (Noyes et al. 1984); however, and as we will see in Sect. 4.4, it is not completely clear if the 24.5-day photometric period is really related with the rotation period of the star.
HD 192263 is $1.99 \mathrm{~km} \mathrm{~s}^{-1}$, we can estimate the orbital inclination angle. Taking the radius of this $\mathrm{K}$ dwarf to be $0.8 R_{\odot}$, the rotational period implies a $v_{\mathrm{eq}} \sim 1.65 \mathrm{~km} \mathrm{~s}^{-1}$, very close to the observed (minimum) value of $1.99 \mathrm{~km} \mathrm{~s}^{-1}$; both numbers are compatible within the errors. In other words, this star is probably seen almost equator-on, and if the stellar rotation axis is perpendicular to the planetary orbital plane, the measured minimum mass of the planet is probably not far from the real mass.

As already discussed in Santos et al. (2000a), the residuals around the fit are a bit higher than usual. For an average $8 \mathrm{~m} \mathrm{~s}^{-1}$ precision for the individual measurements, the measured $12.5 \mathrm{~m} \mathrm{~s}^{-1}$ represent an excess of about $10 \mathrm{~m} \mathrm{~s}^{-1}$. The high 
Table 2. Orbital elements of the fitted orbit and main planetary properties.

\begin{tabular}{lcl}
\hline \hline$P$ & $24.348 \pm 0.005$ & {$[\mathrm{~d}]$} \\
$a_{1} \sin i$ & $0.0203 \pm 0.0004$ & {$[\mathrm{Gm}]$} \\
$T$ & $2451979.28 \pm 0.08$ & {$[\mathrm{~d}]$} \\
$e^{\dagger}$ & 0.0 & \\
$V_{\mathrm{r}}$ & $-10.686 \pm 0.001$ & {$\left[\mathrm{~km} \mathrm{~s}^{-1}\right]$} \\
$\omega^{\dagger}$ & 0.0 & {$[\mathrm{deg}]$} \\
$K_{1}$ & $61 \pm 1$ & {$\left[\mathrm{~m} \mathrm{~s}^{-1}\right]$} \\
$f_{1}(m)$ & $5.64 \pm 0.38$ & {$\left[10^{-10} M_{\odot}\right]$} \\
$\sigma(\mathrm{O}-\mathrm{C})$ & 12.5 & {$\left[\mathrm{~m} \mathrm{~s}^{-1}\right]$} \\
$N$ & 182 & \\
$m_{2} \sin i$ & 0.72 & {$\left[M_{\text {Jup }}\right]$} \\
$a$ & 0.15 & {$[\mathrm{AU}]$} \\
\hline
\end{tabular}

$\dagger$ fixed; when free, $e=0.013 \pm 0.022$, consistent with a circular orbit according to the Lucy \& Sweeney (1971) test.

activity level observed for this $\mathrm{K}$ dwarf could explain at least part of this noise (e.g. Saar et al. 1998; Santos et al. 2000b). Using Eq. (1) in Saar \& Donahue (1997), and considering the stellar $v \sin i$ of $1.99 \mathrm{~km} \mathrm{~s}^{-1}$ and a spot filling factor of about $1 \%$ (as found by Henry et al. 2002), we estimate that the observed radial-velocity variation should have a semi-amplitude of about $12 \mathrm{~m} \mathrm{~s}^{-1}$. We note, however, that this result is some sort of a maximum value, since it is computed for an equatorial spot in a star seen equator-on.

In the lower panel of Fig. 1 we present the residuals of the 24.4-day Keplerian fit. As it can be seen from the plot, there seems to exist a long term trend in the data, with a significant slope of about $4.8 \pm 0.8 \mathrm{~m} \mathrm{~s}^{-1} \mathrm{yr}^{-1}$. The source of this trend is still not clear, and might be due e.g. to the presence of another planetary or stellar companion, or to some long-term activityinduced radial velocity variation connected to a possible stellar magnetic activity cycle (e.g. Kürster et al. 2003). In any case, it is in part responsible for the residuals of the short period fit.

Finally, we have correlated the CORALIE spectra using a Cross-Correlation mask specially constructed for the radialvelocity determination of M4 dwarfs (Delfosse et al. 1998). As seen for HD 41004 (Santos et al. 2002), if the companion to HD 192263 was a low mass star (e.g. an M dwarf) the amplitude of the radial-velocity signal would be dependent of the mask used. The results of our analysis reveal, however, that the fitted orbital parameters always remain unchanged. This confirms that HD 192263 has really a very low mass companion.

\section{Planet or spurious activity signal?}

As seen in the previous section, the radial-velocity periodic signal presented by HD 192263 has remained perfectly constant for the last few years, showing no significant phase or amplitude variations. Although this strongly supports the planetary explanation, a quite similar situation was also found for HD 166435 by Queloz et al. (2001). This latter case and the one presented here are, however, quite different. First, the two stars have different spectral types: late-F and $\mathrm{K}$ dwarf, respectively. Secondly, the rotational velocities are very different; $v \sin i=$ $7.6 \mathrm{~km} \mathrm{~s}^{-1}$ for HD 166435 against $v \sin i=1.99 \mathrm{~km} \mathrm{~s}^{-1}$ for
HD 192263. Furthermore, in our case the fitted "orbit" is perfectly compatible with circular, contrarily to the situation for HD 166435. Finally, in the case of HD 166435, there was a clear correlation between the radial velocity and bisector measurements, not found by Santos et al. (2000a) for HD 192263.

\subsection{Bisector analysis}

The use of the bisector analysis has been shown to be crucial in disentangling planetary signatures from spurious radialvelocity signals (e.g. Queloz et al. 2001; Santos et al. 2002). For HD 192263 this analysis has already been presented in Santos et al. (2000a), revealing no traces of bisector variations related with the radial velocity. This result was considered as a strong evidence for the planetary origin of the radial-velocity signal. However, given the doubts raised by the recent work of Henry et al. (2002), and the much larger number of points available now, it is worth repeating the test.

Using the procedure presented in Queloz et al. (2001) we have computed the Bisector Inverse Slope (BIS) for each of the measured CORALIE cross-correlation functions (CCF's). In Fig. 3 we show the results, plotting the derived values of BIS against the observed radial-velocities (upper panel). The plot shows that there is no evident correlation between the two variables. A Spearman correlation coefficient of 0.18 is obtained. Thus, BIS does not significantly correlate with the radial velocities.

It is important to further note that the observed slope has the opposite sign than in the case of HD 166435, for which an anti-correlation was found. HD 166435 is the only clear published example of activity-induced planetary-like signature. Other cases from the CORALIE planet-search programme are under study (see e.g. Santos et al. 2000c).

In the two lower panels of Fig. 3 we plot a phase-folded diagram of the BIS, constructed using the same period as observed in the radial velocity, as well as the FT of the data. No periodic variation seems present. In particular, we see no sign of variations of BIS with the period of 24 .4-days, as seen in the radial-velocity data. These results strongly support the planetary explanation proposed by Santos et al. (2000a) and Vogt et al. (2000) as the source for the radial-velocity signal.

\subsubsection{Efficiency of the bisector diagnostic for low rotators?}

Using a simple model, we have checked the sensitivity of the bisector analysis to discriminate activity-related radial-velocity variations from real planetary signatures. Our model consists in a stellar disk divided in a grid of $200 \times 200$ cells. Considering that the stellar-template CCF (i.e. a spectral line) in the center of the stellar disk is well approximated by a Gaussian function with given depth and width ${ }^{4}$, we have computed the CCF for each cell. In this process we took into account the cell positions on the disk, to account for the limb-darkening effect (a factor of 0.6 was taken - Gray 1992), and the projected radial

\footnotetext{
${ }^{4}$ values of 0.25 and $4.30 \mathrm{~km} \mathrm{~s}^{-1}$ are respectively chosen as typical values for CORALIE spectra.
} 

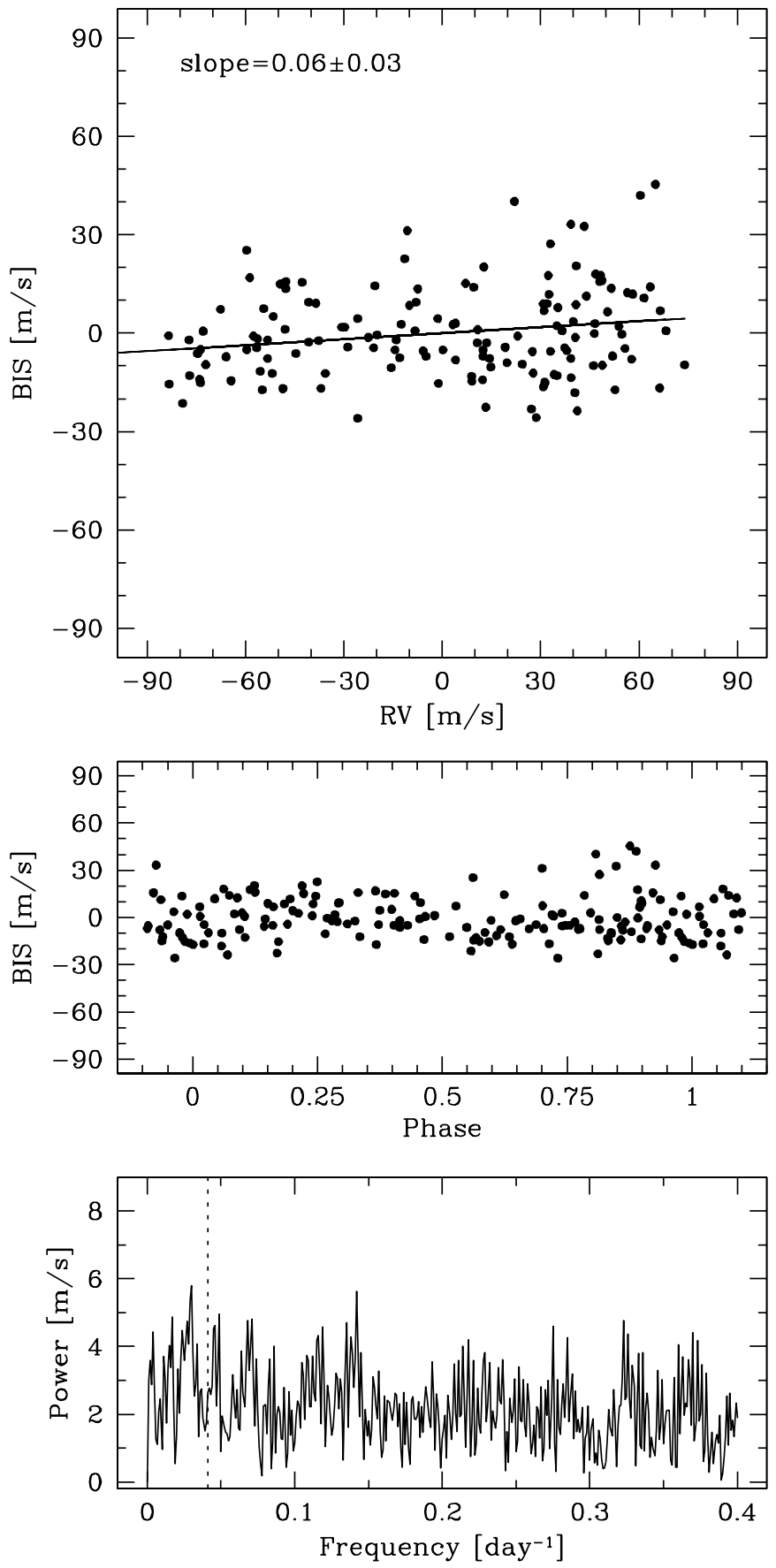

Fig. 3. Upper panel: radial velocity vs. BIS for HD 192263 (as defined in Queloz et al. 2001). The slope and its uncertainty are indicated. The Spearman correlation coefficient between the two variables is 0.18 . Middle panel: BIS values phase folded with the orbital period. Lower panel: FT of the BIS values. No significant period is found in the data. The dotted line is positioned at the period of 24.4-days. Only the best measurements (with errors lower than $10 \mathrm{~m} \mathrm{~s}^{-1}$ ) are considered in these three plots.

velocity. The different CCF's were then added. This procedure was repeated for several "stars" with different $v \sin i$ values (from 0 to $10.0 \mathrm{~km} \mathrm{~s}^{-1}$ ).

From the resulting stellar-disk CCF's, we have subtracted a CCF corresponding to the light that is masked by an equatorial stellar spot positioned at an angle of 65 degrees from the

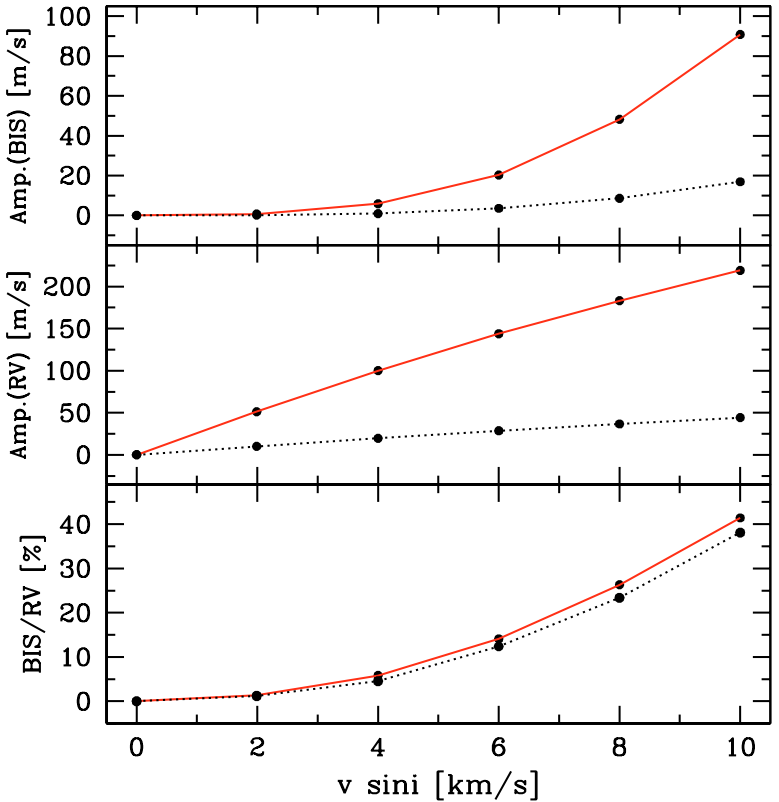

Fig. 4. Upper panels: modeled amplitudes of bisector inverse slope (BIS) and radial velocity, induced by spots with filling factors of $5 \%$ and $1 \%$ (solid and dotted line, respectively), plotted as a function of the star's projected rotational velocity. Lower panel: amplitude ratios (in percent) as a function of $v \sin i$. These results were obtained with a very simple model, and should be considered only as qualitative. See text for more details.

center of the disk (the star is seen equator-on). This value was taken to coincide with the approximate maximum perturbation in radial velocity that a spot might produce (see e.g. Fig. 1 in Saar \& Donahue 1997). Spots with different filling factors were considered as well.

The results of this simple model are presented in Fig. 4 where we plot the radial velocities, bisector "span" (in this case the Bisector Invese Slope, BIS, as defined in Queloz et al. 2001), and the ratio of the BIS-to-radial-velocity amplitudes, as a function of $v \sin i$. We observe that, for low $v \sin i$ values, the influence of a spot on the measured velocity is much larger than the effect observed on the CCF bisector. In the case of HD 192263, with $v \sin i=1.99 \mathrm{~km} \mathrm{~s}^{-1}$, this ratio is around $1-2 \%$. The radial-velocity (semi-)amplitude induced in this low $v \sin i$ model for a spot with a filling factor of $5 \%$ (larger than the one observed in photometry for HD 192263) is around $50 \mathrm{~m} \mathrm{~s}^{-1}$ i.e. similar to the actually observed value. For the same model we find, however, a BIS with an amplitude of only $\sim 1 \mathrm{~m} \mathrm{~s}^{-1}$. Both values are in good agreement with the results obtained from Eqs. (1) and (2) in Saar \& Donahue (1997), attesting the reality of this effect. However, some differences exist since the bisector span definition used by these authors is not the same as the one used here, and probably also due to the simplification of our model.

As it was also shown by Saar \& Donahue (1997), for higher $v \sin i$ models this ratio is higher. The effect is of the order of $\sim 50 \%$ for a $v \sin i=10.0 \mathrm{~km} \mathrm{~s}^{-1}$, and thus clearly detectable. This is mostly due to the higher sensitivity on $v \sin i$ of the effect the spot has onto the bisector when compared with the effect induced on the velocity itself (Saar \& Donahue 1997). 
As shown by Santos et al. (2000c), this qualitative effect is indeed observed, as there seems to exist a clear correlation between the observed BIS-to-radial-velocity amplitude ratios and the stellar projected rotational velocity.

The model described above is, however, far from being perfect. For example, we would obtain a value for the BIS-toradial-velocity ratio of only $\sim 0.2$ in the case of HD 166435 (a late-F dwarf with $v \sin i=7.60 \mathrm{~km} \mathrm{~s}^{-1}$ ), while the observed value is close to 1 (Queloz et al. 2001). The difference is probably due to effects not taken into account, connected e.g. to the geometry of the system or even to the presence of inhomogeneous convection effects - see discussion in Saar \& Donahue (1997). The results obtained here thus probably represent lower limits for the bisector variations. Finally, changes in the spot filling factor do not seem to strongly influence the BIS-toradial-velocity amplitude ratio.

In other words, it is possible that the bisector test is less sensitive for slow rotators, although a small effect should still be visible ${ }^{5}$. But we further caution that this very simple model has its own limitations, and the effects discussed above should thus be seen as a qualitative but not quantitative result. The derived BIS-to-radial-velocity amplitude ratios should not be taken as established values. Finally, these facts do not exclude the planetary explanation for the case of HD 192263, but rather show that for this particular star the bisector test may not be as efficient as we could eventually imagine. Another diagnostic is needed.

\subsection{Photometry}

The results presented in Henry et al. (2002), calling into question the planetary nature of the observed radial-velocity variations, are mostly based on the discovery that HD 192263 has a periodic photometric signal with a period similar to the one observed in radial-velocity. However, Henry et al. (2002) could not directly compare the photometry and the radial-velocities obtained at the same moment in time. This comparison should be done to completely establish a relation between the origin of these two quantities (photometry and radial velocities).

In order to address this problem, while monitoring the radial velocities of HD 192263 with CORALIE, we have started a simultaneous photometric campaign on this star. From May 30 to November 5, 2002, HD 192263 has been measured 187 times in the GENEVA photometric system (Golay 1980) with the photoelectric photometer P7 (Burnet \& Rufener 1979), completely refurbished in 2001 and mounted on the 120-cm Belgian MERCATOR telescope in La Palma (IAC, Canary Islands, Spain). The global photometric reduction procedure is described in Rufener (Rufener 1964, 1985). However, in this particular case, two additional comparison stars, HD 194953 (G8 III) and HD 196712 (B7 III) have been

\footnotetext{
5 Although not the case with the CORALIE spectra $(R=50000)$, we should add that the spectral resolution might also impose limits to the validity of the bisector test. In fact, given that the broadening factors essentially sum up in quadrature, it is very difficult to put in evidence intrinsic line asymmetries when the instrumental profile is significantly broader than the intrinsic line profile.
}
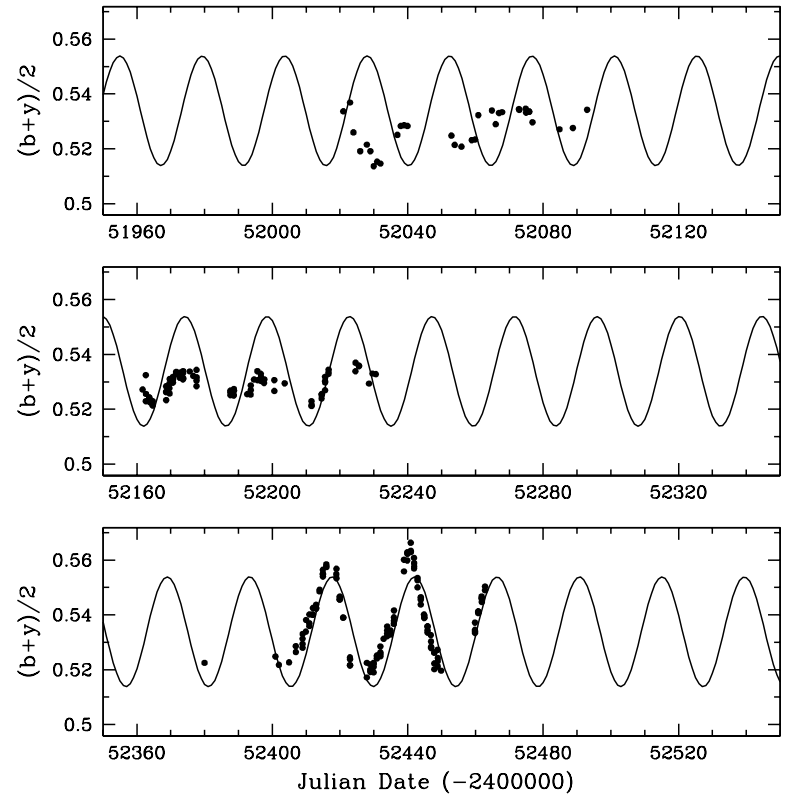

Fig. 5. Photometric measurements of Henry et al. (2002) plotted as a function of time. The three panels correspond to panels 4,5 and 6 of Fig. 2. Superimposed with the photometry is a sinusoidal curve with the same period and phase as the radial-velocity Keplerian fit.

systematically measured together with HD 192263, in order to improve the final data set. The photometric data in the GENEVA system are collected in the General Catalogue (Rufener 1988) and its up-to-date database (Burki et al. 2002). An analysis of the standard stars shows that the final precision of the data is between 0.002 and 0.004 mag $^{6}$.

Finally, we have also used the photometric measurements published in Henry et al. (2002), as we have radial-velocity measurements that coincide (in time) with these data.

A look at the GENEVA data reveals that, indeed, there are clear photometric variation as observed by Henry et al. (2002). However, these variations do not seem to be stable. We shall discuss the different aspects of the question in the following subsections.

\subsection{Correlating the various parameters}

In Fig. 5 we can see a plot of the photometric measurements listed by Henry et al. (2002). These data were used by the authors to show that HD 192263 presents a periodic photometric variation with the same period as the one observed in radial velocity. In the diagram, we have drawn a sinusoidal curve with the same phase and period as the one observed in radialvelocity (same as seen in Fig. 2). The amplitude of this curve

${ }^{6}$ Besides this photometric campaign, C. Nitschelm (private communication) has furnished us a series of photometric measurements of HD 192263 obtained during the last three years at the Danish 0.5-m telescope (La Silla, ESO, Chile). The data show no special photometric variations for this star over the whole period. However, given the obtained precision of only about $0.01 \mathrm{mag}$, these observations cannot be used to strongly constrain our results, and in particular to check for the presence of short period photometric variations. 
was visually set to reproduce the amplitude of the last group of points.

A look at the figure reveals two interesting features. First, for the last measurements, there is a clear superposition of the "radial-velocity" curve and the photometry; the phase and period seems to be about the same. However, for the earlier cycles (two upper panels) this fact is not present. Although the precision of the measurements is the same (as can be seen from the very small dispersion), the amplitude is much smaller. This is, of course, in complete disagreement with the stability of both phase and amplitude observed in the radial velocities. For example, in the period between JD $=2452160$ and 2452200 , for which both RV and photometry exist, the latter looks almost stable while the radial velocities vary with the usual $\left(\sim 60 \mathrm{~m} \mathrm{~s}^{-1}\right)$ amplitude, period, and phase (see Fig. 2). Furthermore, in the upper panel of Fig. 5, it seems that for the region around JD $=2452040$ the phase of the photometric variations has changed.

These considerations already strongly suggest that, during the time of the photometric measurements, either there has been a phase shift in the position of the spot group (eventually disappearing an re-appearing at another location) or else that the stellar rotational period (responsible for the photometric variation) is not exactly the same as the one found in radial velocity. In any case, these observations seem to be contradictory with the idea that the observed radial-velocity variation is being induced (only) by the presence of the spots responsible for the photometric variations.

To further investigate this fact, we plot in Fig. 6 the simultaneous temporal sequencies of GENEVA-photometry observations, CORALIE radial velocities, residuals to the Keplerian 24.4-day fit, and BIS values. In the three upper panels, we have drawn a sinusoidal curve corresponding to the best Keplerian solution derived from the radial-velocity data (see Sect. 3). As previously, for the photometry, the amplitude of this curve was adjusted in order to better fit the data.

The comparison of these plots shows that while the radial velocities follow a very stable (in period, amplitude, and phase) periodic variation, the photometry presents a strange behaviour. At first, we see a clear variation with the same period and phase as the radial velocities. However, from a given moment on, the photometric variations become quite random, and no clear periodicity exists any more. There is even the impression that the relative phase of the photometric and radial-velocity variations is changing slowly, something that could imply e.g. that the rotational period of the star and the radial-velocity periodic signal do not have the very same length. During the whole period, both BIS and residuals are reasonably constant in time. A look at the residuals (mostly for the first group of points) shows, however, what seems to be a small amplitude (about $20 \mathrm{~m} \mathrm{~s}^{-1}$ ) coherent radial-velocity signal left ${ }^{7}$. The same marginal trend is seen for the BIS during this particular time interval for which the photometry shows a clear periodic variation. These trends might be related to the radial-velocity variation induced by the spot group. But the fact that in the global residuals (see Sect. 3)

7 The quality of the data does not permit to precisely access the phase of this periodic signal.

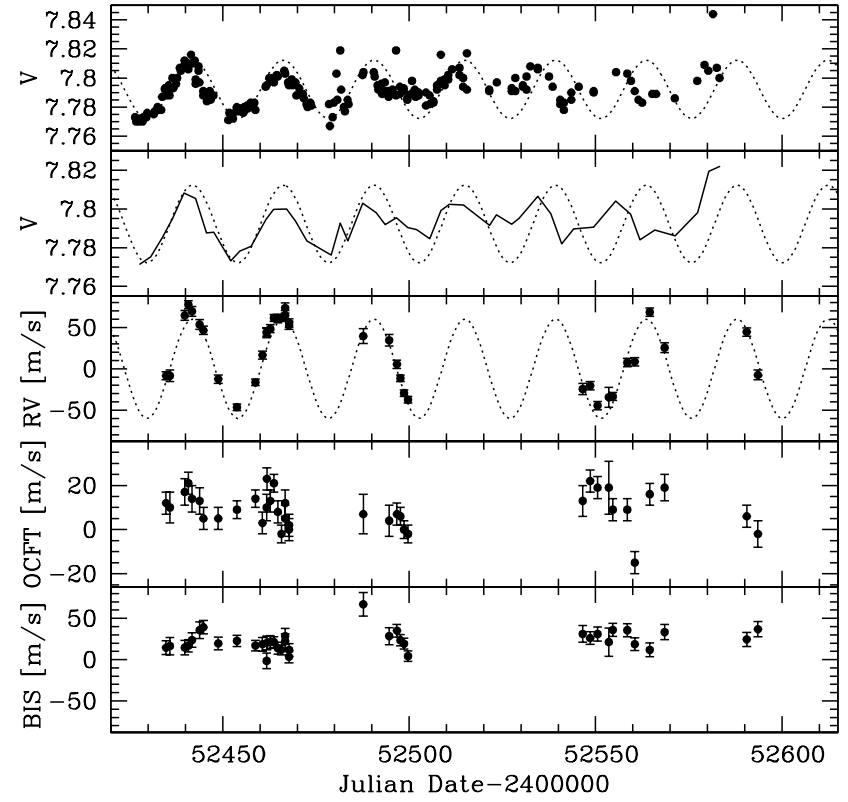

Fig. 6. Plots of the GENEVA-photometry points (upper panel) and 3-day binned photometric data (second panel, solid line), CORALIE radial velocities, residuals to the Keplerian fit (OCFT), and BIS values, as a function of time for the period of simultaneous observations. The sinusoidal curve on top of the velocity points (third panel) illustrates the global Keplerian solution obtained for our data (see Sect. 3). For the two panels with the photometric data, the "fitted" dotted line has the same period and phase as for the radial velocities.

no similar period appears in the Fourier Transform, suggests that this variation is sporadic, as expected since the photometric variations are not stable ${ }^{8}$.

This simultaneous analysis brings many doubts onto the conclusions of Henry et al. (2002). If the presence of spots (and other stellar surface features) were the source for the radialvelocity variations, we should definitely see a correlation between the photometry and the radial-velocity data for the period of our simultaneous measurements. Nothing is seen. Except if we imagine that there are other stellar features not observable in photometry but on the other hand able to induce radial-velocity variations, it is very difficult to accept that the observed stable radial-velocity signal is being caused by activity-related phenomena ${ }^{9}$.

Another interesting detail also deserves some attention. If a spot is responsible for a significant radial-velocity signal, there should be a phase shift between the photometry and the radialvelocity signal. This shift, observed by Queloz et al. (2001) for HD 166435, may be justified by simple considerations. When a (single) spot is at the center of the disk, the photometric

\footnotetext{
${ }^{8}$ We have tried to verify if there was any relation between the radial-velocity residuals and the BIS. Nothing is seen, maybe because of the large errors in the individual measurements, when compared to the magnitude of the effect.

${ }^{9}$ In this context, note also that for $\mathrm{K}$ dwarfs the convective velocities are not very high (smaller than for $\mathrm{F}$ dwarfs), reducing the probability of radial-velocity variations induced by convective inhomogeneities (Saar \& Donahue 1997; Saar et al. 1998; Santos et al. 2000b).
} 
"variation" should be the highest. At the same time, the spot will cause no radial-velocity shift. On the other hand, the maximum effect in radial velocity should happen when the spot is located in an intermediate position between the center of the disk and the limb (e.g. Saar \& Donahue 1997). According to this simple view, the fact that in the first group of points in Fig. 6 we see a clear phase-alignment between the radial-velocity and the photometric measurements, suggests that these two quantities cannot be directly related.

\subsection{Star-planet interactions?}

As seen in the previous sections, the idea that the observed radial-velocity periodic variations are being induced by the presence of photospheric features (e.g. spots) is not satisfying as a whole. In other words, the planetary model, as first discussed in Santos et al. (2000a) and Vogt et al. (2000) should again be considered as the best explanation. Still, the fact that the rotational period of the star seems, according to the photometry, to be similar to the planetary orbital period is intriguing.

One simple way of explaining this is to say that this is pure coincidence, i.e. that the rotational period of the star is by chance similar (but not necessarily equal) to the planetary orbital period. This is not so unrealistic. Known exoplanets have periods from a few days to several years. Most of the stellar low rotators (as e.g. the Sun) have rotational periods of the order of 20 days. They, moreover, form the sample bulk of the programmes searching for planets with the radial-velocity technique. The probability that a $24-d$ period planet fall in this subsample of low rotating star is large. Of course, the closer the photometric and orbital periods, the lower this probability.

Another possibility can also be explored. In the last couple of years a few studies have been published regarding the interaction between the exoplanets and their host stars. As discussed by Cuntz et al. (2000) and Saar \& Cuntz (2001), this interaction might be the result of tidal or/and magnetic effects. These can thus be responsible for observable features such as chromospheric/coronal heating, or chromospheric activity phenomena, possibly inducing changes in the measured radial velocities. A star suffering strong magnetic interactions with the planet could e.g. have a hot spot rotating with the same period as the planet. On the other hand, tidal interactions could induce variations with half the orbital period. In this context, Rubenstein \& Schaefer (2000) have proposed that the observation of "superflares" might be related to the presence of close-in giant planets. Recently, Shkolnik et al. $(2002,2003)$ have found evidences that a few stars known to harbour close-in planetary companions present important activity induced effects, observable as variations in the $\mathrm{Ca}$ II $\mathrm{H} \& \mathrm{~K}$ line reversals. Based on this discovery, we suggest that the photometric variability observed for HD 192263 might very well be the result of such kind of star-planet interactions.

As discussed in Sect. 4.3, there is no offset in phase between the radial-velocity and the photometric variations (over the period of time during which the two persist). This shift means that the spot is at the "center" of the disk (and unable to induce radial-velocity variations) when the planet is producing the maximum radial-velocity variation (i.e. when it is located at phase 0.0 ). In other words, the spot is not located at the subplanetary point, but rather at an angle of about 90 degrees. This fact could be seen a signature that the spot (or spot group) is due to tidal friction effects, and that the delay is due to a process similar to the ones producing the phase shift between the position of the moon and the maximum tidal height on earth ${ }^{10}$ (or to some other physical process causing a delay in the reaction of the stellar photosphere/chromosphere to the perturbations). Such an offset was also possibly found by Shkolnik et al. (2002) for $v$ And. If true, this could also mean that the system is not synchronized; as we have seen in the previous sections, this would not be a surprise, since there might be a continuous phase variation between the radial-velocity and photometric signals. It is further interesting to note that a period half of the orbital one is seen in the Mount Wilson S index (Henry et al. 2002), a possible value if the effects observed are of tidal origin (Cuntz et al. 2000).

However, the current data do not give us the possibility to exclude any of the possibilities (tidal, magnetic, or others). In the case of a magnetic origin for the interaction, this would give us the possibility to access the magnetic field of the planet, which would be, of course, an interesting result.

\section{Conclusions}

We have presented the case of HD 192263, a star that shows very stable periodic radial-velocity variations. While these were first considered as the signature of a planetary companion, this interpretation was recently called back into question by the detection of periodic photometric variations with a period very close to the orbital period (Henry et al. 2002). In order to understand the true source of the observed radial-velocity signal, we have gathered precise photometry, radial-velocity, and bisector measurements for this star, part of them taken simultaneously. Our results are the followings:

- The radial-velocity variations show a striking long-term stability in period, phase, and amplitude. A very different scenario is found for the photometry that alternates moments of "stability" with periods of variability. Cross-correlation BIS (Bisector Inverse Slope) measurements do not correlate either with radial velocities. These observations very strongly supports the idea that photometry and radial-velocity variations do not share the same origin. Except if some unknown photospheric phenomenon is being responsible for the observed radial-velocity variation without influencing the stellar photometric behaviour in the same manner, the $\sim 24$.4-day period in the radial-velocity data remains best interpreted by the presence of a low-mass planet around HD 192263.

- The similarity of the measured radial-velocity and photometric periods can be interpreted in several ways. On the one hand, it can be a simple coincidence. On the other hand, it can be the result of interactions (magnetic or tidal) between

\footnotetext{
10 It should be noted, however, that the timescales of the tidal motions in the low-density stellar atmosphere are short (Cuntz et al. 2000).
} 
the planet and the star, and able to induce activity-related phenomena.

- The radial-velocity data also show the possible signature of a long-term trend. The nature of this trend is not known. Possible explanations might involve the presence of another planetary (or stellar) companion, or even long term activityinduced radial velocity variations. A clear followup of this result will be possible with instruments like HARPS (Pepe et al. 2002a).

This paper shows that the use of photometry as a tool to confirm (or not) the presence of the planetary mass companions to solar-type stars, detected by radial-velocity technique, should be taken cautiously. The results should always be analyzed carefully, and whenever possible, the various data (radial-velocities, photometry, bisector) should be obtained simultaneously in time.

Acknowledgements. Nuno Santos dedicates this work to the memory of Gustavo Camejo Rodrigues, who will always be dearly remembered. We would like to thank Tim Brown for fruitful discussions, C. Nitschelm, A. Vidal-Madjar, and A. Lecavelier for having provided us with photometric measurements of HD 192263, and the numerous observers at the MERCATOR telescope, from Leuven and Geneva, for the successful photometric monitoring. We wish to thank the Swiss National Science Foundation (Swiss NSF) for the continuous support to this project. Support from Fundação para a Ciência e Tecnologia, Portugal, to N.C.S., in the form of a scholarship, is gratefully acknowledged.

\section{References}

Brown, T. M., Kotak, R., Horner, S., et al. 1998, ApJS, 117, 563 Burki, G., et al. 2002, http: //obswww . unige .ch/gcpd/ph13.html Burnet, M., \& Rufener, F. 1979, A\&A, 74, 54

Cuntz, M., Saar, S. H., \& Musielak, Z. E. 2000, ApJ, 533, L151

Delfosse, X., Forveille, T., Mayor, M., et al. 1998, A\&A, 338, L67

Flower, P. J. 1996, ApJ, 469, 355

Golay, M. 1980, Vistas Astron., 24, 141

Gray, D. 1992, in The observation and analysis of stellar photospheres (Cambridge Univ. Press)

Gray, D. F. 1997, Nature, 385, 795

Gray, D. F. 1998, Nature, 391, 153

Halbwachs, J.-L., Arenou, F., Mayor, M., Udry, S., \& Queloz, D. 2000, A\&A, 355, 573

Han, I., Black, D., \& Gatewood, G. 2001, ApJ, 548, L57
Henry, G. W., Donahue, R. A., \& Baliunas, S. L. 2002, ApJ, 577, L111

Jorissen, Mayor, M., \& Udry, S. 2001, A\&A, 379, 992

Kürster, M., Endl, M., Rouesnel, F., et al. 2003, A\&A, 403, 1077

Lucy, L. B., \& Sweeney, M. A. 1971, AJ, 76, 544

Mayor, M., \& Queloz, D. 1995, Nature, 378, 355

Noyes, R. W., Hartmann, L. W., Baliunas, S. L., Duncan, D. K., \& Vaughan, A. H. 1984, ApJ, 279, 763

Paulson, D. B., Saar, S. H., Cochran, W. D., \& Hatzes, A. P. 2002, AJ, 124,572

Pepe, F., Mayor, M., Rupprecht, G., et al. 2002a, The Messenger, 110, 9

Pepe, F., Mayor, M., Galland, F., et al. 2002b, A\&A, 388, 632

Pourbaix, D. 2001, A\&A, 369, L22

Pourbaix, D., \& Arenou, F. 2001, A\&A, 372, 935

Queloz, D., Henry, G. W., Sivan, J. P., et al. 2001, A\&A, 379, 279

Rubenstein, E. P., \& Schaefer, B. E. 2000, ApJ, 529, 1031

Rufener, F. 1964, Publ. Obs. Genève, A, 66, 413

Rufener, F. 1985, in Calibration of Fundamental Stellar Quantities, ed. D. S. Hayes et al. (Dordrecht: Reidel Publ. Co.), IAU Symp., 111, 253

Rufener, F. 1988, Geneva Photometric Catalogue, 4th ed., Obs. Genève

Saar, S. H., \& Cuntz, M. 2001, MNRAS, 325, 55

Saar, S. H., \& Donahue, R. A. 1997, ApJ, 485, 319

Saar, S. H., Butler, R. P., \& Marcy, G. W. 1998, ApJ, 498, L153

Santos, N. C., Mayor, M., Naef, D., et al. 2000a, A\&A, 356, 599

Santos, N. C., Mayor, M., Naef, D., et al. 2000b, A\&A, 361, 265

Santos, N. C., Mayor, M., Naef, D., et al. 2000c, in Proc. IAU 202 Symp. on Planetary Systems in the Universe: Observations, Formation, and Evolution, ed. A. Penny, P. Artimowicz, A.-M. Lagrange, \& S. Russel, ASP Conf. Ser., in press [astro-ph/0101377]

Santos, N. C., Mayor, M., Naef, D., et al. 2002, A\&A, 392, 215

Santos, N. C., Israelian, G., Mayor, M., Rebolo, R., \& Udry, S. 2003 , A\&A, 398, 363

Shkolnik, E., Walker, G. A. H., \& Bohlender, D. A. 2002, AAS, 201.4616 [astro-ph/0209014]

Shkolnik, E., Walker, G. A. H., \& Bohlender, D. A. 2003, ApJ, submitted [astro-ph/0303557]

Tinney, C. G., McCarthy, C., Jones, H. R. A., et al. 2002, MNRAS, 332,759

Udry, S., Mayor, M., Naef, D., et al. 2000, A\&A, 356, 590

Vogt, S. S., Marcy, G. W., Butler, R. P., \& Apps, K. 2000, ApJ, 536, 902

Zucker, S., Mazeh, T., Santos, N. C., Udry, S., \& Mayor, M. 2003, A\&A, 404, 775 